\title{
A randomised, prospective study comparing trabeculectomy with viscocanalostomy with adjunctive antimetabolite usage for the management of open angle glaucoma uncontrolled by medical therapy
}

\author{
D P S O'Brart, M Shiew, B Edmunds
}

Br J Ophthalmol 2004;88:1012-1017. doi: 10.1136/bjo.2003.037432

\begin{abstract}
Aims: To compare trabeculectomy with viscocanalostomy augmented with adjunctive antimetabolite use for the control of intraocular pressure (IOP) in open angle glaucoma (OAG).

Methods: 45 patients (50 eyes) with uncontrolled OAG were randomised to either trabeculectomy (25 eyes) or a viscocanalostomy technique (25 eyes). Preoperatively, all eyes were graded in terms of risk factors for drainage failure and were given intraoperative antimetabolites (5-fluorouracil $25 \mathrm{mg} / \mathrm{ml}$ (5-FU), mitomycin C (MMC) $0.2 \mathrm{mg} / \mathrm{ml}$ and $0.4 \mathrm{mg} / \mathrm{ml}$ ) according to a standard protocol.

Results: There were no significant differences between the groups in age, sex, type of OAG, preoperative medications, risk factors for drainage failure, and preoperative IOP. Mean follow up was 20 months (range 3-24 months). It was 12 months or longer in all eyes, except two lost to follow up at 3 months. At 12 months, complete success (IOP $<21 \mathrm{~mm} \mathrm{Hg}$ without antiglaucoma medications) was seen in $91 \%$ of eyes undergoing trabeculectomy, but in only $60 \%$ of eyes undergoing viscocanalostomy $(p<0.02)$. Similarly, at the last follow up visit (mean 20 months) complete success was seen in $68 \%$ of eyes undergoing trabeculectomy and $34 \%$ with viscocanalostomy $(p<0.05)$. In terms of qualified success (IOP $<21 \mathrm{~mm} \mathrm{Hg}$ with or without glaucoma medications) and mean IOP measurements postoperatively there were no difference between the groups, although the mean number of antiglaucomatous medications required postoperatively was less with trabeculectomy (0.39) than viscocanalostomy (1.04) $(p<0.05)$. Needling procedures were more commonly required after trabeculectomy $(p<0.02)$. YAG goniotomy was required in three eyes (13\%) after viscocanalostomy. Early transient complications such as anterior chamber shallowing and encysted blebs were more common in the trabeculectomy group $(p<0.05)$. Late postoperative cataract formation was similar between the two groups.

Conclusion: In terms of complete success and number of antiglaucomatous medications required postoperatively, IOP control appears to be better with trabeculectomy. Viscocanalostomy is associated with fewer early transient postoperative complications.
\end{abstract}

$\mathrm{T}$ abeculectomy has been the filtration procedure of choice for the past 30 years. ${ }^{12}$ It is quick, technically easy to perform, has fewer complications than full thickness procedures, and high reported success rates. ${ }^{3-9}$ However, early and late filtration failures are not infrequent, especially in eyes with risk factors for drainage failure, ${ }^{10-14}$ and sight threatening complications occasionally occur. ${ }^{1-9}$ To optimise results, frequent and careful outpatient monitoring is required; interventions such as suture release, lysis, and needling are not uncommon; and the adjunctive use of antimetabolites, with their associated problems, is now standard practice especially in eyes with risk factors for failure. ${ }^{15-17}$

Such limitations have led to the investigation of alternative drainage techniques, including "non-penetrating" procedures. These surgeries involve deep scleral dissection beneath a superficial scleral flap with deroofing of Schlemm's canal and opening of the canal with viscoelastics (viscocanalostomy) $^{18}$ or insertion of a collagen implant (deep sclerectomy). ${ }^{19} 20$ Such techniques avoid intraocular penetration, possibly reducing overdrainage or the risk of endophthalmitis. Peripheral iridectomy is not required, lessening the breakdown of the blood-aqueous barrier, potentially resulting in less anterior chamber inflammation with perhaps fewer cataracts, synechiae, and bleb failure and possibly a reduced need for antimetabolites. In addition, the mechanism of aqueous filtration may not rely entirely on the subconjunctival route, but may occur via Schlemm's canal and/or increased uveoscleral outflow.

Initial clinical results of non-penetrating techniques have been encouraging, in that reported postoperative complications have been few. ${ }^{18}{ }^{21-31}$ However, success rates have been variable. Some have reported success rates comparable to trabeculectomy, ${ }^{181-26}$ while others have been somewhat less favourable. ${ }^{27-31}$ In most publications, eyes with risk factors for drainage failure have been largely excluded and further procedures such as neodymium:yttrium-aluminium-garnet (Nd:YAG) laser goniopuncture have been required to maintain drainage in a high proportion of eyes. Thus far there have been only a few studies comparing non-penetrating techniques with trabeculectomy. While some have suggested similar outcomes in terms of drainage success, ${ }^{32-34}$ most have demonstrated more effective intraocular pressure (IOP) control with trabeculectomy. ${ }^{35-40}$ We have previously published the results of a prospective, randomised study, reporting better IOP control with trabeculectomy compared to viscocanalostomy. ${ }^{40}$ In this study, intraoperative antimetabolites were used according to a standardised protocol for eyes with risk factors for drainage failure undergoing trabeculectomy, as this is standard practice. ${ }^{10-14}$ They were not used (similar to previous published studies) in eyes undergoing viscocanalostomy, as it has been postulated that such techniques may reduce the need for antimetabolites and may

Abbreviations: 5-FU, 5-fluorouracil; IOP, intraocular pressure; MMC, mitomycin $C ;$ OAG, open angle glaucoma 
not rely on the subconjunctival route for aqueous drainage. Careful examination of the eyes in our study suggested that subconjunctival drainage is an important outflow pathway in viscocanalostomy. Therefore, more favourable results might have been achieved with viscocanalostomy and differences in IOP control perhaps nullified, if antimetabolites had been used as an adjunct in both operative groups. In order to investigate this, we conducted a further randomised, controlled study in which antimetabolites were used according to a standardised protocol in eyes undergoing both trabeculectomy and viscocanalostomy.

\section{PATIENTS AND METHODS}

Following ethics committee approval, 45 patients (50 eyes) with uncontrolled open angle glaucoma (OAG) were randomised, between June 2000 and May 2001, to either trabeculectomy (25 eyes) or a viscocanalostomy technique (25 eyes). Randomisation was performed using a sealed envelope system, where 50 shuffled envelopes designating the surgery to either trabeculectomy or viscocanalostomy, were opened before surgery. Patient demographic data are shown in table 1. Before entry into the study, consent was obtained from all patients. Inclusion criteria for the study were primary or secondary open angle glaucoma uncontrolled on maximally tolerated medical therapy. Exclusion criteria were congenital glaucoma and any type of angle closure glaucoma. Patients were not excluded from the study on the basis of their age, race, previous ocular surgery, or any other risk factor for drainage failure.

Preoperatively full baseline data were obtained for each patient and included a full ocular and medical history, visual field assessment (Humphery 24-2 computerised perimetry), slit lamp biomicroscopy, Goldmann applanation tonometry, gonioscopy, and mydriatic funduscopy.

Based on a standardised protocol (table 2), all eyes were graded in terms of their risk factors for drainage failure. On the basis of their protocol score, eyes undergoing trabeculectomy were selected to receive intraoperative antimetabolites (5-fluoruracil $25 \mathrm{mg} / \mathrm{ml}$ (5-FU), mitomycin C (MMC) $0.2 \mathrm{mg} / \mathrm{ml}$ and $0.4 \mathrm{mg} / \mathrm{ml}$ ) (table 2 ).

\section{Surgical techniques}

Immediately preoperatively single applications of pilocarpine $4 \%$, amethocaine $1 \%$, and chloramphenicol $0.5 \%$ drops were instilled into the operative eye. A single surgeon (DO'B), using retrobulbar anaesthesia with $2 \%$ lignocaine, performed all surgeries. Following insertion of a lid speculum, a 7/0 silk traction suture was inserted at the inferior corneal-scleral limbus if required.
Table 2 Protocol for antimetabolite use and risk factor for failure scoring (Based on protocol developed by Professor RN Weinreb, Shiley Eye Centre, San Diego)

\begin{tabular}{ll}
\hline Risk factors for failure & Score \\
\hline Race: Indian, Asian & 3 \\
Afro-Caribbean & 5 \\
Combined cataract and glaucoma surgery & 5 \\
Previous ocular surgery & 5 \\
Secondary glaucoma (uveitis, neovascular) & 5 \\
Topical treatments $>2$ & 3 \\
Duration of topical treatment $>1$ year & 3 \\
IOP target $<16$ mm Hg & 3 \\
Conjunctival cicatrising disease & 5 \\
Age $<20$ years & 5 \\
Age $<40$ years & 3 \\
Diabetes & 1 \\
Previous ALT & 1 \\
\hline
\end{tabular}

If score less than 5: give no antimetabolites; between 6-10: give 5-FU $(25 \mathrm{mg} / \mathrm{ml})$; between 11-15: give MMC $0.2 \mathrm{mg} / \mathrm{ml}$; over 15: give MMC $0.4 \mathrm{mg} / \mathrm{ml}$.

\section{Trabeculectomy}

Trabeculectomy was performed via a fornix based conjunctival flap. If readily apparent, Tenon's capsule was excised. Haemostasis was achieved using bipolar cautery. Antimetabolites were applied using a single cellulose sponge (John Weiss, UK). This was soaked in the appropriate antimetabolite and fashioned into an approximate $2.0 \times 4.0 \mathrm{~mm}$ rectangular shape and applied to the scleral bed and subconjunctival space taking care to avoid exposure to the conjunctival wound edge. Application time was 5 minutes for 5-FU and 2 minutes for MMC. During the application time the cellulose sponge was moved using forceps over the scleral bed for 2-3 clock hours, 1-2 mm behind the limbus in order to afford a large area of antimetabolite application. Following irrigation with balanced saline solution, a $3.0 \mathrm{~mm}$, triangular shaped, one third thickness scleral flap was fashioned to within $0.5 \mathrm{~mm}$ of the limbus. A crescent blade was used to tunnel into clear cornea. A paracentesis was performed 90 degrees from the trabeculectomy site. The anterior chamber entered just beyond the limbus using a $3.2 \mathrm{~mm}$ slit blade. A punch trabeculectomy was performed using a crozeform punch (Altomed, UK). A peripheral iridectomy was fashioned and the scleral flap closed with three 10/0 nylon sutures, two at the base near the limbus and one at the apex. Releasable sutures were not used. The surgeon adjusted the tension of the sutures, based on the depth of the anterior chamber, an estimation of intraocular pressure by palpation (after reformation of the anterior chamber with an injection of balanced saline

\begin{tabular}{|lll|}
\hline \multicolumn{2}{l}{ Table 1 Preoperative patient } & demographics \\
\hline Characteristic & Trabeculectomy $(\mathbf{n}=\mathbf{2 5})$ & Viscocanalostomy ( $\mathbf{n}=\mathbf{2 5}$ ) \\
\hline Age (years) & 59 (range 20-79) & 61 (range 28-80) \\
Sex (male:female) & $18: 7$ & $17: 8$ \\
Race: & & 8 \\
White & 8 & 15 \\
Afro-Caribbean/African & 14 & 2 \\
Indian Subcontinent & 3 & 2.87 (range 1-4) \\
Preoperative glaucoma medications & 3 (range 2-5) & 8.9 (range (3-16) \\
Risk factors for failure score (table 2) & 9.1 (range 3-16) & 23 \\
Type of OAG: & 24 & 2 \\
Primary & 1 & 25.7 (18-44) \\
Secondary & 27.9 (range 19-50) & \\
Preoperative IOP (mm Hg) & & \\
\hline
\end{tabular}


solution through a paracentesis) and the degree of fluid egress under the scleral flap. The conjunctiva was closed with a continuous $8 / 0$ Vicryl suture.

\section{Viscocanalostomy}

Viscocanalostomy was performed using a procedure similar to that described by Stegmann et al, ${ }^{18}$ who had personally instructed the surgeon (DO'B) in the technique. Before the study the surgeon had been performing the procedure for over 24 months. A fornix based conjunctival flap was fashioned and if apparent, Tenon's capsule was excised. Haemostasis was achieved using bipolar cautery. Antimetabolites were applied using a single cellulose sponge (John Weiss, UK) to the scleral bed and subconjunctival space, using the technique described above. Following antimetabolite application and irrigation, a $5.0 \mathrm{~mm}$ triangular shaped, one third thickness scleral flap was fashioned and dissected 1.0 to $2.0 \mathrm{~mm}$ into clear cornea. A second triangular flap was dissected $0.5 \mathrm{~mm}$ inside the border of the first. This deeper flap constituted approximately two thirds of scleral thickness, leaving only a thin translucent layer overlying the choroid. With forward dissection of this flap, Schlemm's canal could be identified approximately $1.0 \mathrm{~mm}$ posterior to the limbus and deroofed. A moistened cellulose sponge was used to apply gentle pressure on Schwalbe's line to separate Descemet's membrane from the overlying stroma and create an intact Descemet's "window", at least $1.0 \mathrm{~mm}$ in width, though which aqueous could diffuse. The deep scleral flap was excised at its base using Vannas scissors. A specially designed cannula (Grieshaber, Switzerland), with an outer diameter of $150 \mu \mathrm{m}$, was introduced into the ostia of Schlemm's canal, left and right, to inject high viscosity sodium hyaluronate (Healonid GV, Pharmacia, UK) into the canal in an attempt to widen its diameter. The superficial scleral flap was closed with three 10/0 nylon sutures, two at the base near the limbus and one at the apex, using the technique describe above. Healonid GV was injected under this flap into the space created by removal of the deep flap. The conjunctiva was closed with a continuous 8/0 Vicryl suture.

\section{Postoperative management}

Immediately postoperatively a subconjunctival injection of Betnesol and cefuroxime was given, a drop of phenylepherine $10 \%$ instilled and the eye padded overnight. Postoperatively, topical chloramphenicol $0.5 \%$ was administered three times a day for 2 weeks and prednisolone $1 \%$ eight times a day for 2 weeks; this was then reduced over the next 3 months.

Postoperatively patients were examined at day 1 and then at 1, 2, 4, and 8 weeks and 3, 6, 12, 18, and 24 months. At each visit a full ocular examination was performed including slit lamp biomicroscopy, Goldmann applanation tonometry, gonioscopy, and mydriatic funduscopy. In addition, visual field assessment was performed at 6, 12, 18, and 24 months.

Needling procedures with subsequent subconjunctival injections of 5-FU were performed on eyes in both operative groups with drainage failure due to encysted blebs, where just the conjunctiva was penetrated or excessive subconjunctival fibrosis, where an attempt was made to lift the scleral flap. Great care was taken in eyes after viscocanalostomy to ensure that penetration into the anterior chamber, converting the procedure to full thickness, did not occur during needling.

Nd:YAG goniotomy was performed in selected eyes after Viscocanalostomy with drainage failure and unsatisfactory IOP control during the first 3 months after surgery.

\section{Statistical methods}

Student's $t$ tests were used to compare continuous variables between the groups such as IOP differences. $\chi^{2}$ analysis was used to compare qualitative data. Results with $\mathrm{p}<0.05$ were considered statistically significant.

\section{RESULTS}

\section{Patient demographics}

There were no significant differences in terms of age, sex, race, preoperative IOP, numbers of preoperative glaucoma medications, type of open angle glaucoma, and risk factors for failure score between eyes undergoing trabeculectomy and viscocanalostomy (table 1 ).

\section{Operative data}

Viscocanolostomy generally took longer to perform, average time of first incision to closure 31 minutes (range 2040 minutes), than trabeculectomy, average time of first incision to closure 21.6 minutes (range 15-35 minutes) $(\mathrm{p}<0.01)$.

Two eyes $(8 \%)$ undergoing viscocanalostomy were converted to a trabeculectomy at the time of surgery, owing to rupture of Descemet's membrane during surgery. Careful observation of loss of convexity and an increase in the egress of fluid through the apparently intact Descemet's window suggested the presence of small perforations in 10 eyes (40\%).

\section{Postoperative interventions}

Needling procedures were more common after trabeculectomy and were performed in 16 eyes $(64 \%)$, compared to six eyes $(23 \%)$ after viscocanalostomy $(\mathrm{p}>0.02)$.

Three eyes (13\%) with early drainage failure after viscocanalostomy underwent Nd:YAG goniotomy in the first 3 months after surgery. In two of these eyes, following the laser procedure, IOP control was improved and has thus far been maintained below $21 \mathrm{~mm} \mathrm{Hg}$ without antiglaucomatous medications.

\section{Intraocular pressure control}

The mean follow up was 20 months (range 3-24 months) and was 12 months or longer in all eyes, except two lost to follow up at 3 months.

In all groups immediately postoperatively IOP was significantly reduced. The mean IOP in the trabeculectomy group was $7 \mathrm{~mm} \mathrm{Hg}$ (range $0-15 \mathrm{~mm} \mathrm{Hg}$, median $7 \mathrm{~mm} \mathrm{Hg}$ ) at $\mathrm{l}$ day and $7.88 \mathrm{~mm} \mathrm{Hg}$ (range $1-18 \mathrm{~mm} \mathrm{Hg}$, median $6 \mathrm{~mm} \mathrm{Hg}$ ) at 1 week, compared to $4.82 \mathrm{~mm} \mathrm{Hg}$ (range 0$15 \mathrm{~mm} \mathrm{Hg}$, median $4 \mathrm{~mm} \mathrm{Hg}$ ) at $\mathrm{l}$ day and $8.65 \mathrm{~mm} \mathrm{Hg}$ (range $0-20 \mathrm{mmHg}$, median $8 \mathrm{~mm} \mathrm{Hg}$ ) at 1 week in the viscocanalostomy group. At 1 week 13 eyes (52\%) after trabeculectomy were hypotonous (IOP $<6 \mathrm{~mm} \mathrm{Hg}$ ), compared to 10 eyes $(43 \%)$ after viscocanalostomy. Thereafter, IOP returned to more normal levels with no hypotonous eyes.

At 12 months the percentage of eyes with successful drainage, defined as an IOP of $21 \mathrm{~mm} \mathrm{Hg}$ or less without antiglaucomatous medications, was $61 \%$ with viscocanalostomy and $91 \%$ with trabeculectomy $(\mathrm{p}>0.02)$. At the last follow up visit (mean 20 months) complete success was seen in $34 \%$ of eyes after viscocanalostomy and $68 \%$ after trabeculectomy $(\mathrm{p}>0.05)$. In all viscocanalostomy eyes with successful drainage a subconjunctival drainage bleb was evident. With the exception of a single eye after trabeculectomy with a thin walled, but non-leaking, cystic bleb, the morphology of the drainage blebs after viscocanalostomy and trabeculectomy in cases with successful drainage looked very similar, with diffuse posterior blebs, albeit with central areas of avascularity, being evident.

The percentage of eyes achieving an IOP of $15 \mathrm{~mm} \mathrm{Hg}$ or less without antiglaucomatous medications at the last follow up visit (mean 20 months), was 44\% (11 eyes) with trabeculectomy and 17\% (four eyes) with viscocanalostomy. 


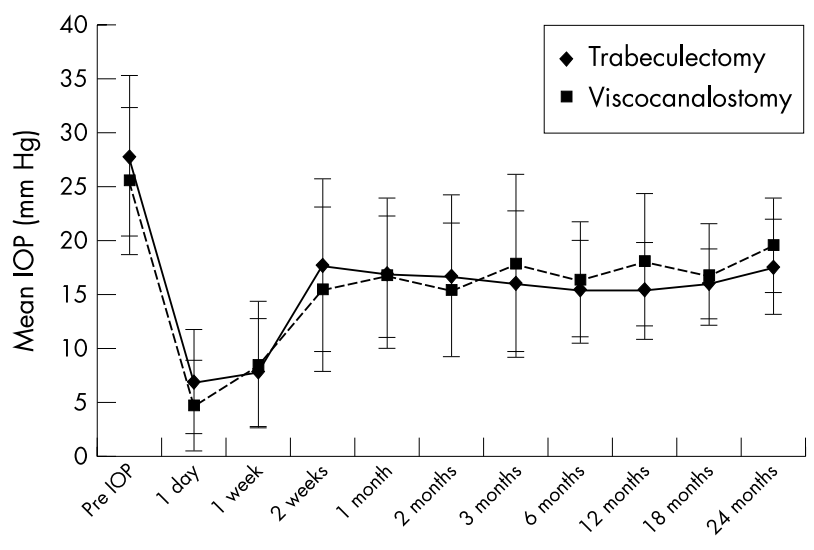

Figure 1 Mean intraocular pressure (IOP) with time. Comparison of trabeculectomy $(n=25)$ with viscocanalostomy $(n=23)$.

The IOP results for the first 24 months are summarised in figure 1 . There were no statistically significant differences in terms of mean IOP between the two operative groups at any time postoperatively. With a mean follow up of 20 months, six eyes $(24 \%)$ in the trabeculectomy group are receiving antiglaucomatous medication to maintain an intraocular pressure below $21 \mathrm{~mm} \mathrm{Hg}$ compared to 13 eyes (57\%) in the viscocanalostomy group $(\mathrm{p}<0.02)$. At the last follow up visit the average number of antiglaucomatous medications required postoperatively was 0.36 for the trabeculectomy and 1.04 for the viscocanalostomy patients $(p<0.05)$. Two eyes in the trabeculectomy group $(8 \%)$ and five eyes in the

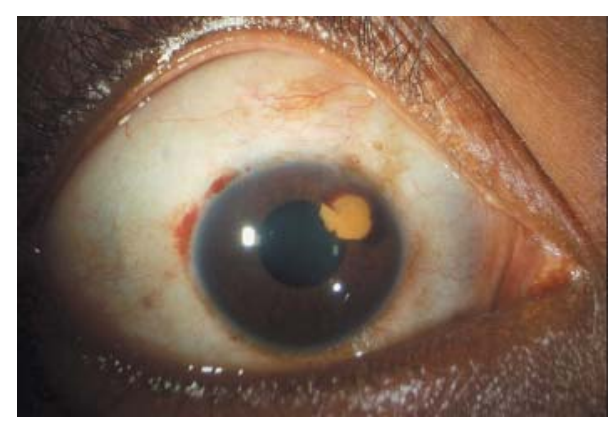

Figure 2 Photograph of a localised area of altered intracorneal haemorrhage at the level of Descemet's membrane 6 months after viscocanalostomy. viscocanalostomy group (22\%) have undergone further glaucoma drainage surgery (trabeculectomy) owing to complete failure of drainage and inadequate IOP control on antiglaucomatous medications. Following this second procedure, intraocular pressure control has been satisfactory without the need for antiglaucomatous medications in all seven eyes. The two eyes in the viscocanalostomy group, which were converted to trabeculectomy, have successful drainage with good IOP control, requiring no antiglaucomatous medications thus far, with a follow up of 24 months.

\section{Complications}

Postoperative complications are detailed in table 3. Early complications such as transient bleb leaks $(\mathrm{p}<0.05)$ and transient anterior chamber shallowing $(\mathrm{p}<0.05)$ were more common after trabeculectomy. The occurrence of encysted blebs were more common after trabeculectomy $(p<0.05)$. In one patient who underwent viscocanalostomy, a localised area of intracorneal haemorrhage at the level of descemet's membrane, $3 \mathrm{~mm}$ in diameter, was seen adjacent to the drainage site immediately postoperatively (fig 2 ). This are did not reach the visual axis and has slowly cleared with time although remnants of altered blood are still evident 2 years postoperatively. In one eye after trabeculectomy a thin walled cystic bleb (with no aqueous leakage) was present. There were no overhanging or excessively large blebs in either group (table 3 ).

Cataract formation, occurring between 6-18 months postoperatively was similar between the two groups, with three eyes in each group requiring surgery (table 3 ). These six eyes have all undergone successful phacoemulsifiaction cataract surgery with return of preoperative Snellen visual acuity.

\section{DISCUSSION}

The results of this prospective, randomised study indicate that trabeculectomy is superior to viscocanalostomy for the control of IOP in open angle glaucoma. Although there were little differences in terms of mean IOP levels postoperatively between the two treatment groups, complete success rates (IOP $<21 \mathrm{~mm} \mathrm{Hg}$ with no antiglaucomatous medications) at 12 months and the last follow up visit were significantly better with trabeculectomy. In addition, the number of eyes receiving antiglaucomatous medications and the average number of medications required postoperatively in order to maintain an IOP $<21 \mathrm{~mm} \mathrm{Hg}$ were significantly less with trabeculectomy.

Review of the current literature largely supports these findings. While some have suggested similar outcomes between trabeculectomy and non-penetrating techniques in

Table 3 Early and late complications after trabeculectomy and viscocanalostomy

\begin{tabular}{lll}
\hline & Trabeculectomy & Viscocanalostomy \\
\hline Early complications & $4(16 \%)$ & $1(4 \%)$ \\
Transient Bleb Leak & $11(44 \%)$ & $7(30 \%)$ \\
Transient hyphaemia & $8(32 \%)$ & $2(9 \%)$ \\
Transient anterior chamber shallowing & $6(24 \%)$ & $4(17 \%)$ \\
Injected blebs & $7(28 \%)$ & $1(4 \%)$ \\
Encysted blebs & $1(4 \%)$ & $1(4 \%)$ \\
Transient overlarge blebs & 0 & 0 \\
IOP spike & $1(4 \%)$ & $1(4 \%)$ \\
Peripheral anterior synechiae & 0 & $1(4 \%)$ \\
Intracorneal haemorrhage & $1(4 \%)$ & 0 \\
Late complications & 0 & 0 \\
Cystic blebs & 0 & 0 \\
Overlarge/overhanging blebs & 0 & 0 \\
Hypotony (IOP<6 mm g) & $3(12 \%)$ & $3(13 \%)$ \\
Late bleb leak & & \\
Patients requiring cataract surgery & &
\end{tabular}


terms of drainage success, ${ }^{32-34}$ most studies have demonstrated more effective IOP control with trabeculectomy. ${ }^{35-40}$ Of the three papers suggesting similar efficacy of IOP control, two were non-randomised with only retrospective analysis of eyes undergoing trabeculectomy, ${ }^{32}{ }^{34}$ and while significant differences were not demonstrated between the treatment groups, the general trend was for better IOP control with trabeculectomy. In addition, the use of Nd:YAG goniopuncture to augment drainage (converting eyes to a fully penetrating technique), the avoidance of intraoperative antimetabolites (considered standard practice in trabeculectomy in eyes with risk factors for failure ${ }^{15-17}$ ), and the exclusion of eyes with risk factors for drainage failure, may have biased the results in these studies in favour of nonpenetrating techniques. Of the comparative studies in the literature demonstrating more effective IOP control with trabeculectomy all are well constructed, randomised bilateral or prospective studies. ${ }^{35-40}$ Five compared trabeculectomy with viscocanalostomy and one was a comparative study of deep sclerectomy. ${ }^{35}$ These six studies demonstrated superior results in terms of IOP control with trabeculectomy albeit with fewer early postoperative complications with nonpenetrating techniques. ${ }^{35-40}$

Previously, we published the results of a randomised, prospective study, reporting better IOP control with trabeculectomy. ${ }^{40}$ In this study intraoperative antimetabolites were used for eyes undergoing trabeculectomy, but not for viscocanalostomy, as it had been postulated that such techniques reduced the need for antimetabolites and did not rely on the subconjunctival route for aqueous drainage. ${ }^{18-31}$ However, careful postoperative examination of our patients suggested that subconjunctival drainage was indeed an important outflow pathway in viscocanalostomy. ${ }^{40}$ We therefore proposed that more favourable results might have been achieved with viscocanalostomy and differences in IOP control perhaps nullified, if antimetabolites had been used as an adjunct in both operative groups. Certainly other investigators have reported improved success rates with the use of MMC in eyes undergoing non-penetrating glaucoma drainage techniques. ${ }^{41}$ In order to investigate the relative importance of adjunctive antimetabolite use in viscocanalostomy, the current study was designed with antimetabolites being used according to a standardised protocol (table 2 ) in both treatment groups. However, despite the use of antimetabolites, our results still indicate that viscocanaolostomy is inferior to trabeculectomy in terms of IOP control. These findings are supported by those of Kobayahi et al, ${ }^{39}$ who in a randomised bilateral controlled study, found better IOP control with trabeculectomy than viscocanalostomy despite the use of MMC in both groups.

The mechanism of aqueous drainage in non-penetrating trabecular techniques is uncertain. Authors have postulated that drainage may be either subconjunctival, through Schlemm's canal, via increased uveoscleral outflow or by a combination of these pathways. ${ }^{18-31}$ Careful postoperative examination of our patients in this and our previous study, ${ }^{40}$ demonstrated that in all eyes with successful drainage after viscocanalostomy evidence of subconjunctival drainage of aqueous was apparent, confirmed by the presence of conjunctival epithelial microcysts and drainage blebs. In eyes without successful drainage these changes were not evident. This would support the hypothesis that the subconjunctival route is the main drainage pathway in viscocanalostomy. However, if this were the case, one might expect the results of the current study, where antimetabolites were used to limit subconjunctival fibrosis in both trabeculectomy and viscocanalostomy, to demonstrate similar outcomes in terms of IOP control. However, this is not the case and our results and those of Kobayahi et al ${ }^{39}$ clearly demonstrate that trabeculectomy appears to offer more effective subconjunctival drainage of aqueous, despite adjunctive antimetabolite use. This perhaps indicates an important role for a penetrating or "complete" fistula into the anterior chamber in the maintenance of subconjunctival aqueous drainage after glaucoma filtration surgery. This factor may especially important in the maintenance of long term drainage. It is of interest that in this and our previous study, ${ }^{40}$ the results of viscocanalostomy at 6 months were very encouraging, with successful drainage in over $90 \%$ of eyes and comparable results to trabeculectomy. With further follow up, however, the results became disappointing, with only $60 \%$ of eyes undergoing viscocanalostomy achieving successful drainage at 12 months and even fewer at 18 months. While it is difficult to explain the mechanisms responsible for failure of drainage after 6 months, other investigators have documented late drainage failure with non-penetrating techniques. ${ }^{27}$ The observation of the disappearance of subconjunctival blebs in our patients after viscocanalostomy, suggests that continuing subconjunctival fibrosis is responsible for late drainage failure. The presence of a fully penetrating fistula in trabeculectomy with relatively high flow, compared to a nonpenetrating, low flow fistula in viscocanalostomy, may perhaps have a protective effect mechanically preventing fibrotic closure of the subconjunctival space, thus preventing late drainage failure. If a "high flow," fully penetrating fistula is important in the maintenance of subconjunctival drainage after glaucoma filtration surgery, then there may perhaps be a role for early (within the first 3 months) $\mathrm{Nd}$ :YAG goniotomy in all eyes after non-penetrating glaucoma drainage techniques. This requires further investigation, although it must be remembered that such interventions increase the risk of postoperative hypotony, especially with the adjunctive use of antimetabolites.

It is of interest that with the exception of one eye after trabeculectomy with a thin walled cystic bleb, the morphology of the drainage blebs in both viscocanalostomy and trabeculectomy was similar. In virtually all eyes will successful drainage, diffuse posterior blebs, albeit with central areas of avascularity, were evident. In no eyes were there large overhanging or thin walled, leaking cystic blebs, despite the extensive use of antimetabolites. All eyes in our study underwent drainage surgery using fornix based conjunctival flaps. In addition, a large surface area (2-3 clock hours) of scleral and subconjunctival tissue was exposed to antimetabolite during application. We believe that such techniques reduced the risk of cystic bleb formation in our study patients for both trabeculectomy and viscocanalostomy procedures. In a recent study Wells et $a l^{42}$ have reported a reduced risk of cystic bleb formation with fornix versus limbus based conjunctival flaps. These results, together with the observations in our study, suggest that factors concerning conjunctival flap formation are more important in the development of cystic blebs than penetrating versus non-penetrating drainage techniques.

As in our previous study, ${ }^{40}$ early postoperative complications such as anterior chamber shallowing and bleb leaks were less common after viscocanalostomy, although such problems were transient and non-sight threatening. Encysted blebs and the requirement for needling procedures postoperatively were more commonly encountered after trabeculectomy. This is in contrast to our previous study. ${ }^{40}$ While this is not ideal, as such procedures can, albeit very rarely, be associated with ocular morbidity, none of our patients suffered any adverse events following needling and successful drainage was maintained in the majority of eyes. Interestingly, in cataract formation was similar between the two treatment groups. This once again is in contrast with our previous study, ${ }^{40}$ where cataract formation was more 
commonly encountered after trabeculectomy, and may in some way perhaps reflect the use of antimetabolites in both groups in the current study.

Although viscocanalostomy offers some theoretical advantages, in terms of its less invasive nature and possible reduced reliance on subconjunctival drainage, intraocular pressure control despite the use of adjunctive antimetabolites, appears to be superior with trabeculectomy and it continues to be the filtering procedure of choice for the management of glaucoma. Viscocanalostomy is generally associated with fewer early postoperative complications, but significant problems permanently impairing vision did not occur with either technique.

\section{Authors' affiliations}

D P S O'Brart, M Shiew, B Edmunds, Department of Ophthalmology, St Thomas's Hospital, London SEl 7EH, UK

Correspondence to: Mr D P S O'Brart, Department of Ophthalmology, St Thomas's Hospital, London SE1 7EH, UK; davidobrart@aol.com

Accepted for publication 30 December 2003

\section{REFERENCES}

1 Sugar HS. Experimental trabeculectomy in glaucoma. Am J Ophthalmol $1961 ; 51: 623$

2 Cairns JE. Trabeculectomy. Preliminary report of a new method. Am J Ophthalmol 1968;66:673-9.

3 Cains JE. Surgical treatment of primary open-angle glaucoma. Trans Ophthalmol Soc UK 1972;92:745-56.

4 Watson PG. Surgery of the glaucomas. Br J Ophthalmol 1972;56:299.

5 Watson PG, Barrett F. Effectiveness of trabeculectomy in glaucoma. Am J Ophthalmol 1975;79:831-45.

6 Wilson P. Trabeculectomy: long-term follow-up. Br J Ophthalmol 1977;61:535-8

7 Shields MB. Trabeculectomy versus full-thickness filtering operations for control of glaucoma. Ophthalmic Surg 1980;11:498-505.

8 Zaidi AA. Trabeculectomy: a review and 4-year follow-up. $\mathrm{Br} J$ Ophthalmol 1980;64:436-9.

9 Mills KB. Trabeculectomy: a retrospective long-term follow-up of 444 cases. Br J Ophthalmol 1981;65:790-5.

10 Egbert PR, Williams AS, Singh K, et al. A prospective trial of intraoperative fluorouracil during trabeculectomy in a black population. Am J Ophthalmol 1993; 116:612-16.

11 Gressel MG, Heuer DK, Parrish RK. Trabeculectomy in young patients. Ophthalmology 1984;91:1242-6.

12 Broadway DC, Grierson I, Hitchings RA. Local effects of previous conjunctival incisional surgery and the subsequent outcome of filtration surgery. Am J Ophthalmol 1998; 125:805-8.

13 Gross RL, Feldman RM, Spaeth GL, et al. Surgical therapy of chronic glaucoma in aphakia and pseudophakia. Ophthalmology 1988;95: 1195-201.

14 Allen RC, Bellows AR, Hutchinson BR, et al. Filtration surgery in the treatment of neovascular glaucoma. Ophthalmology 1982;89:1181-7

15 The Fluorouracil Filtering Surgery Study Group. Fluorouracil filtering surgery study one year follow up. Am J Ophthalmol 1989;108:625-35.

16 Chen C-W, Huang H-T, Bair JS, et al. Trabeculectomy with simultaneous topical application of mitomycin-C in refractory glaucoma. J Ocul Pharmacol 1990;6: 175-82.

17 Khaw PT. Antimetabolites in glaucoma filtration surgery. Current medical literature, the Royal Society of Medicine 1996;6:71-7.

18 Stegmann R, Pienaar A, Miller D. Viscocanalostomy for open-angle glaucoma in black African patients. J Cataract Refract Surg 199, 25:316-22.
19 Fyodorov SN, Kozlov VI, Timoshkina NT, et al. Non-penetrating deep sclerectomy in open angle glaucoma. IRTC Eye Microsurgery. Moscow: RSFSR Ministry of Public Health, 1989:52-5.

20 Kozlov VI, Bagrov SN, Anisimova SY, et al. Non-penetrating deep sclerectomy with collagen. IRTC Eye Microsurgery. Moscow: RSFSR Ministry of Public Health, 1989:44-6.

21 Demailly $\mathbf{P}$, Jeanteur-Lunel $M N$, Berkani $M$, et al. La sclerectomie profonde non perforante associee a la pose d'un implant de collagene dans le glaucoma primitif a angle ouvert. J Fr Ophtalmol 1996;19:659-66.

22 Demailly P, Lavat P Kretz G, Jeanteur-Lunel MN. Non-penetrating deep sclerectomy (NPDS) with or without collagen device (CD) in primary openangle glaucoma: middle-term retrospective study. Int Ophthalmol 1997;20:131-40.

23 Sanchez E, Schnyder CC, Sickenberg M, et al. Deep sclerectomy: results with and without collagen implant. Int Ophthalmol 1997;20:157-62.

24 Welsh NH, Delange J, Wasserman P, et al. The "de-roofing" of Schlemm's canal in pateints with open angle glaucoma through placement of a collagen drainage device. Ophthalmic Surg Lasers 1998;29:216-26.

25 Massey J, Gruber D, Muraine M, et al. La sclerectomie profonde non perforante dans le traitement chirurgical du glaucome chrinique a angle ouvert. Resultats a moyen terme. J Fr Ophtalmol 1999;22:292-8.

26 Carassa RG, Bettin P, Fiori $M$, et al. Viscocanalostomy: a pilot study. Eur J Ophthalmol 1998;8:57-61

27 Karlen ME, Sanchez E Schnyder CC, et al. Deep sclerectomy with collagen implant: medium term results. Br J Ophthalmol 1999:83:6-11.

28 Hamard P, Plaza L, Kopel J, et al. Sclerectomie profonde non perforante (SPNP) et glaucome a angle ouvert. Resultants a moyen terme des premiers patients operes. J Fr Ophthalmol 1999;22:25-31.

29 Shaarawy T, Karlen M, Schnyder C, et al. Five-year results of deep sclerectomy with collagen implant. J Cataract Refract Surg 2001;27:1770-8.

30 Drusedau $M$, von Wolff K, Bull H, et al. Viscocanalostomy for primary open angle glaucoma: The Gross Pankow experience. I Cataract Refract Surg 2000;26:1367-73.

31 Sinaric-Megevand G, Levenberger PM. Results of viscocanalostomy for primary open angle glaucoma. Am J Ophthalmol 2001;132:221-8.

32 Mermoud, Schnyder CC, Sickenberg M, et al. Comparison of deep sclerectomy with collagen implant and trabeculectomy in open angle glaucoma. J Cataract Refract Surg 1999;25:323-31.

33 El Sayyad F, Helal M, El-Kholify H, et al. Non-penetrating deep sclerectomy versus trabeculectomy in bilateral primary open angle glaucoma. Ophthalmology 2000;107:1671-4.

34 Ambresin A, Shaarawy T, Mermoud A. Deep sclerectomy with collagen implant in one eye compared with trabeculectomy in the other eye of the same patient. J Glaucoma 2002;11:214-20.

35 Chiselita D. Non-penetrating deep sclerectomy versus trabeculectomy in primary open angle glaucoma surgery. Eye 2001;15:197-201.

36 Janescu-Cuypers C, Jacobi P, Konen W, et al. Primary viscocanalostomy versus trabeculectomy in white patients in open-angle glaucoma: a randomised clinical trial. Ophthalmology 2001;108:254-8.

37 Cassara RG, Bettin P, Foiori $M$, et al. Viscocanalostomy versus trabeculectomy inj white adults affected by open-angle glaucoma: a 2-year randomized, controlled trial. Ophthalmology 2003;110:882-7.

38 Luke C, Dietlein TS, Jacobi PC, et al. A prospective randomized trial of viscocanalostomy versus trabeculectomy in open-angle glaucoma: a 1-year follow-up study. J Glaucoma 2002;11:294-9.

39 Kobayashi H, Kobayashi K, Okinami S. A comparison of the intraocular pressure-lowering effect and safety of viscocanalostomy and trabeculectomy with mitomycin C in bilateral open-angle glaucoma. Graefes Arch Clin Exp Ophthalmol 2003;241:359-66.

40 O'Brart DPS, Rowlands E, Islam N, et al. A randomised, prospective study comparing trabeculectomy augmented with antimetabolites with a viscocanalostomy technique for the management of open angle glaucoma uncontrolled by medical therapy. Br J Ophthalmol 2002;86:748-54.

41 Kozobolis VP, Christodoulakis EV, Tzanakis N, et al. Primary deep sclerectomy versus primary deep sclerectomy with the use of mitomycin $C$ in primary open angle glaucoma. J Glaucoma 2002;11:287-93.

42 Wells AP, Cordeiro MF, Bunce $C$, et al. Cystic bleb formation and related complications in limbus- versus fornix-based conjunctival flaps in pediatric and young adult trabeculectomy with mitomycin C. Ophthalmology 2003;110:2192-7. 